\title{
State of mental healthcare systems in Eastern Europe: do we really understand what is going on?
}

\author{
Dzmitry Krupchanka ${ }^{1}$ and Petr Winkler ${ }^{2}$
}

'Senior Researcher, Department of Social Psychiatry, National Institute of Mental Health, Kecany, Czech Republich, Kecany, Czech Republic, email dzmitry.krupchanka@nudz.cz ${ }^{2}$ Head, Department of Social Psychiatry, National Institute of Mental Health, Klecany, Czech Republic
The article examines the current state of mental healthcare systems in countries of Eastern Europe and derives implications for future research and service development. Analysis of available statistics from the World Health Organization's Mental Health Atlas suggests the need for better-quality data collection. Nonetheless, there appear to be insufficient resources allocated to mental health, lack of involvement of service users in policy-making and, to a large extent, systems continue to rely on mental hospitals. Based on the data presented, a set of directions for future reforms was drafted.

Eastern Europe occupies a large part of the European continent. According to the United Nations' definition which we adhere to in the current paper, Eastern Europe consists of 10 countries: Belarus, Bulgaria, Czech Republic, Hungary, Republic of Moldova, Poland, Romania, Russian Federation, Slovakia and Ukraine. It has a total population of nearly 300 million (approximately 150 million without the Russian Federation) (data from http://esa.un.org/unpd/wpp).

The recent fast development of the global mental health movement has increased the attention given to low- and middle-income countries. However, Eastern Europe has been somewhat overlooked by mental health research and practical initiatives (Forsman et al, 2014). This is despite the burden of mental and behavioural disorders in the region being one of the highest in the world according to the Global Burden of Disease Study (Lozano et al, 2012). According to the World Health Organization (WHO), suicide rates are particularly high (WHO, 2012) and the level of alcohol consumption is enormous and growing (WHO, 2014a).

Eastern Europe went through a political transformation in the 1990s. After the collapse of communism 25 years ago, the countries diverged, pursuing their own paths to development, as well as with respect to the reorganisation and modernisation of their mental healthcare systems. Many positive changes have been achieved but many challenges remain (Füredi et al, 2006; van Voren et al, 2007; Semrau et al, 2011; Dlouhy, 2014).

\section{Methods}

The WHO Mental Health Atlas (WHO-MHA) 2011 and 2014 (WHO, 2011, 2014b) and the European Health For All database were consulted.
After initial screening, we opted to use only the WHO-MHA database, as it seems to be the only one covering details of mental health service functioning. We derived data on basic statistics available for the majority of Eastern Europe countries. Where data were unavailable in the WHO-MHA 2014, we referred to the 2011 database to make comparisons possible.

To analyse trends in institutional care between 2011 and 2014 we have calculated percentage changes in the numbers of in-patient care facilities and mental hospital beds. For the sake of comparison, we extracted data for the countries of Eastern Europe and 15 member states of the European Union (EU) before its enlargement in 2004 (EU15).

\section{Results}

General information and data on resources for mental health and institutional care are presented in Table 1 .

\section{General information}

The majority of Eastern European countries have mental health policies: $77 \%$ of countries (compared with $93 \%$ in the EU15). However, the involvement of service users and families in the policy-making is markedly worse than in the EU15: there is not a single Eastern European country with full participation of users in policy-making, in contrast to $53.5 \%$ of EU15 countries.

\section{Resources for mental health}

In both Eastern Europe and the EU15, the proportion of total health expenditure allocated to mental health $(3.3 \%$ and $7 \%$ respectively) mismatches the global burden of disease for neuropsychiatric disorders $(21.22 \%$ and $29.96 \%$ in terms of disability-adjusted life years). However, this difference is especially poignant in Eastern Europe as the total health expenditure per person is almost six times lower than in the EU15 (US $\$ 758.78$ versus US\$4382.53) and the part allocated to mental health is two times lower (3.3\% versus $7 \%$ ). As a result, the total mental health expenditure per capita is only US $\$ 18.7$, which is 15.7 times lower than in the EU15 (US\$293.7).

The number of overall mental health staff in Eastern Europe is 2.4 times lower than in the EU15 (47.4 versus 127.2 per 100000 ) and there are 1.8 times fewer psychiatrists $(8.03$ versus 14.13 per $100000)$. No Eastern European country reaches the EU15 average level of mental health staff. 


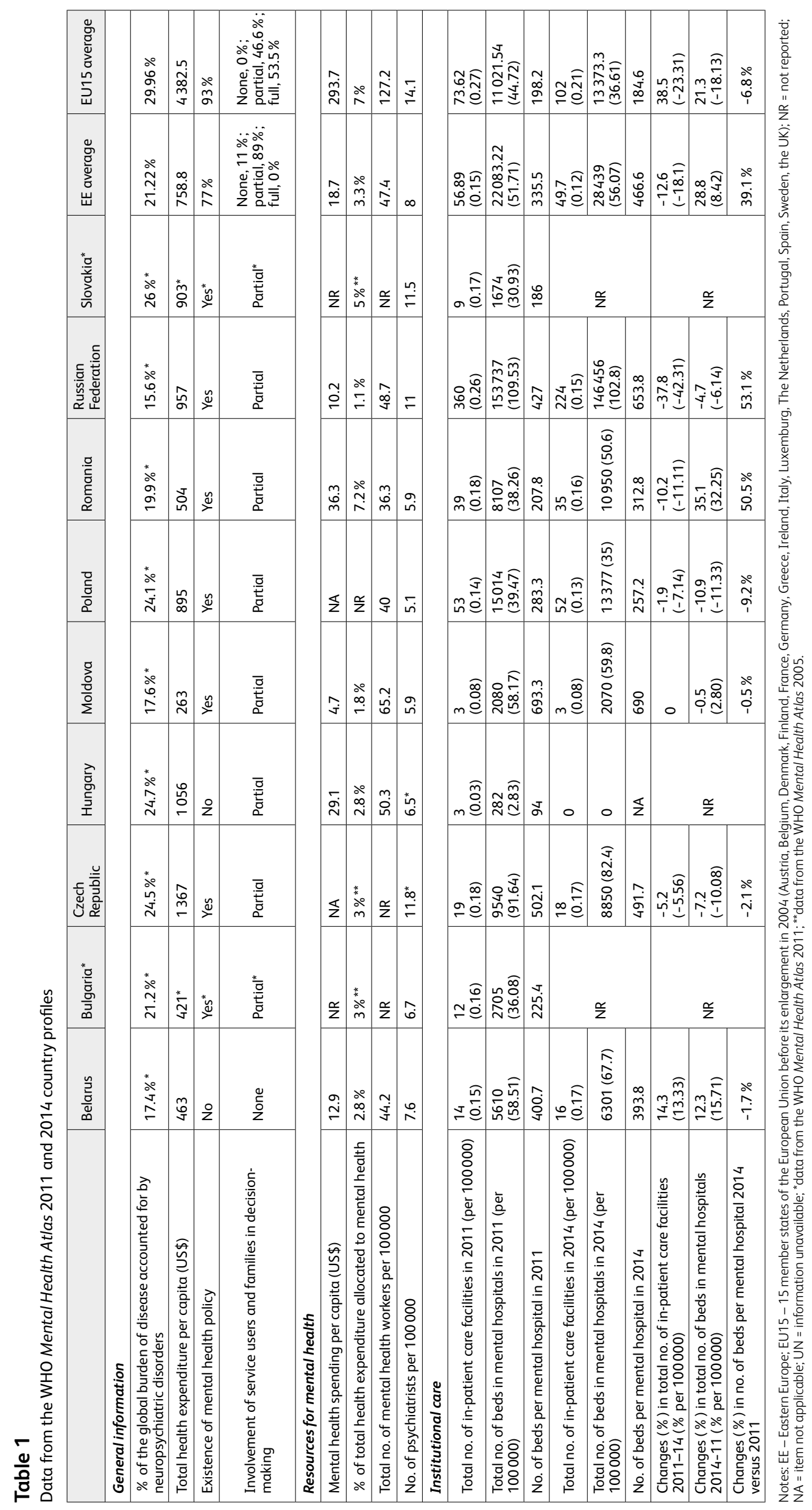




\section{Institutional care}

Although the average number of mental hospitals in countries of Eastern Europe in 2014 was half that in the EU15 (0.12 versus 0.21 per 100000$)$, the number of beds in these facilities was high (53.7 versus 45.6 per 100000 ), as the mental hospitals are considerably larger, with 466.6 beds on average, which is 2.5 times more than in EU15. The largest average number of beds per hospital was found in Moldova (690), followed by the Russian Federation (653.8). In contrast, Hungary did not report the existence of a single mental hospital in 2014.

Between 2011 and 2014, despite the decrease in number of hospitals in Eastern Europe $(-18.1 \%$ per 100000), the number of beds in institutional care facilities increased by $8.4 \%$ per 100000 . The substantial rise in the number of beds per hospital (39.1\%) differed from the EU15, where the number had fallen by $6.8 \%$. This is largely explained by the growth of mental hospitals in the Russian Federation (53.1\% of beds per hospital) and Romania (50.5\%). In contrast, other Eastern European countries slightly decreased the number of beds per hospital and Hungary had discontinued the provision of care within mental hospitals.

\section{Discussion}

The data on mental healthcare systems in Eastern Europe should be treated with caution and with careful consideration of the limitations. Although it was declared that WHO-MHA represents the most accurate and complete information on national mental health service provision available (Taylor Salisbury et al, 2016), the quality of the data is relatively low, and this substantially limits the possibility of a comprehensive overview.

First, the WHO-MHA data were collected without sufficient standardisation of country reports and specification of definitions. Moreover, the absence of the quality control in data collection could increase the chance of poor or even opportunistic data reports. Therefore, between-country comparisons based on the WHO-MHA may be misleading.

Second, WHO-MHA 2005, 2011 and 2014 are, in fact, three different surveys with differences in the formulation of questions and the process of data collection. Therefore, the comparisons of data over time may be prone to bias.

Third, some countries failed to report important pieces of information (e.g. on human resources or community care) and sometimes even whole country reports were missing (e.g. Ukraine).

Finally, databases hardly allow for an assessment of the quality of indicators. For example, despite the policy and legislation reported as existing in the majority of countries, we are unable to assess their quality and feasibility.

In summary, the limitations that we have faced in the current analysis may reflect a general situation of insufficient evidence in the region. This is an obstacle to evidence-based mental healthcare development, as well as to advocacy campaigns and reforms. We see an urgent need to obtain reliable data on the functioning and development of mental healthcare systems in the region in order to better understand what is really going on.

Taking all of these limitations into account, the current analysis poses a number of questions requiring further exploration. First, there is evidence of insufficiency of resources allocated to mental health across the region when considering the burden of neuropsychiatric disorders. Second, despite the multiple calls for deinstitutionalisation (Füredi et al, 2006; van Voren et al, 2007; Semrau et al, 2011; Dlouhy, 2014), mental healthcare in the majority of Eastern European countries seems to continue its reliance on big psychiatric institutions.

Based on the data, a set of directions for future service development in the countries of Eastern Europe may be suggested. First, the limitations of the databases speak in favour of more comprehensive data collection and analysis. Second, insufficient involvement of service users and their families in policy- and decision-making requires redress, so that their views can be better represented and their needs addressed. Third, lack of resources (both financial and human) for mental health must be carefully reconsidered to increase their allocation, to bring them in line with the contribution of mental disorders to the global burden of disease. Furthermore, the process of deinstitutionalisation should be enhanced in most countries of the region, as only minor improvement has been achieved so far. Closing mental hospitals without redirecting resources to community care may result in an increase in the number of beds per hospital remaining. Instead, the transfer of resources into community care and general hospitals should be implemented and carefully monitored in order to avoid the pitfalls of reforms (Saraceno et al, 2007).

However, a number of barriers may hamper progress in the above-mentioned directions: (1) lack of leadership and capacity; (2) disempowerment and weakness of service users and advocacy organisations; (3) stigma as well as lack of awareness of the need for change and an absence of adequate understanding of evidence-based service development and mental health economics on the part of decision-makers; (4) insufficiency of finances; (5) the stability and rigidity of systems and structures largely inherited from communist times; (6) the influence of corruption.

\section{Conclusions}

Despite the slight progress in some Eastern European countries, the development of mental healthcare in the region remains slow and not very effective. The following processes should be facilitated: (1) building capacity and leadership to implement extensive analysis of services and to lead future reforms; (2) collaborative efforts to carefully plan and monitor implementation of mental health policies and legislation; (3) empowerment of service users and their inclusion in decision- and policy-making at all levels; (4) allocation of sufficient resources and their proper distribution to support redirection of services from hospitals to 
the community. Strengthening the human rights approach, organisations for young psychiatrists, international collaboration and additional attention to the region are among the possible solutions.

\section{References}

Dlouhy, M. (2014) Mental health policy in Eastern Europe: a comparative analysis of seven mental health systems. BMC Health Services Research, 14, 42

Forsman, A. K., Ventus, D. B. J., van der Feltz-Cornelis, C. M., et al (2014) Public mental health research in Europe: a systematic mapping for the ROAMER project. European Journal of Public Health, 24, 955-960.

Füredi, J., Mohr, P., Swingler, D., et al (2006) Psychiatry in selected countries of Central and Eastern Europe: an overview of the current situation. Acta Psychiatrica Scandinavica, 114, 223-231.

Lozano, R., Naghavi, M., Foreman, K., et al (2012) Global and regional mortality from 235 causes of death for 20 age groups in 1990 and 2010: a systematic analysis for the Global Burden of Disease Study 2010. Lancet, 380, 2095-2128.

Saraceno, B., van Ommeren, M., Batniji, R., et al (2007) Barriers to improvement of mental health services in low-income and middleincome countries. Lancet, 370, 1164-1174.
Semrau, M., Barley, E. A., Law, A., et al (2011) Lessons learned in developing community mental health care in Europe. World Psychiatry, 10, 217-225.

Taylor Salisbury, T., Killaspy, H. \& King, M. (2016) An international comparison of the deinstitutionalisation of mental health care: development and findings of the Mental Health Services Deinstitutionalisation Measure (MENDit). BMC Psychiatry, 16, 54. Available at http://www.pubmedcentral.nih.gov/articlerender.fcgi?artid=4772656 \&tool=pmcentrez\&rendertype=abstract (accessed 30 May 2016).

van Voren, R., Tomov, T., Keukens, R., et al (2007) Mental health policy and practice across Europe. In Mental Health Policy and Practice Across Europe (eds M. Knapp, et al), pp. 397-425. Open University Press.

WHO (2011) Mental Health Atlas - 2011 country profiles. Available at http://www.who.int/mental_health/evidence/atlas/profiles/en/ (accessed 11 February 2016).

WHO (2012) Suicide data. Available at http://www.who.int/mental health/prevention/suicide/suicideprevent/en/WHO (accessed 4 April 2015).

WHO (2014a) Global Status Report on Alcohol and Health 2014 Available at http://www.who.int/substance abuse/publications/ global_alcohol_report/en (accessed 4 April 2015).

WHO (2014b) Mental Health Atlas - 2014 country profiles. Available at http://www.who.int/mental_health/evidence/atlas/ profiles-2014/en (accessed 11 February 2016)

\title{
Hysterical stupor or yogic sleep?
} The conundrum

\author{
Manohar Dhadphale
}

Academic Head, Kamala Nehru Hospital, Pune, India, email manohar_dhadphale@yahoo. co.uk

\begin{abstract}
A woman in the care of the author 40 years ago was reported to have been sleeping for 2 days. We treated her condition as conversion hysteria. Her private psychiatrist was the renowned R. D. Laing; he was unhappy with our line of management, on the grounds of the arbitrariness of the diagnosis, the labelling of the woman with a diagnosis of hysteria and the treatment of the patient without her consent. In retrospect, I wonder if she was in a state of yogic sleep (yoga nidra).
\end{abstract}

After practising for more than a dozen years (1958-70) in rural and remote parts of Kenya, then known as the Colony and Protectorate, I came to England to train as a psychiatrist. My first clinical posting was in a rapidly changing Victorian mental hospital in south-west London (which closed down decades ago). In my practice at home in rural Kenya, the diagnosis of hysteria was made mainly by excluding a physical basis for the patient's symptoms. I had seen and treated many patients with fits, bizarre and vague symptoms such as abdominal pain, heat in the forehead and dudus (insects) crawling all over the back or legs at certain times of the day. As a general practitioner in the African countryside in the 1960s and 1970s, the treatment was purely symptomatic and supportive.
I came to the UK to train as a psychiatrist and return to Africa after training. Here, I describe an interesting and memorable experience. As a thirdyear trainee, I came face to face with a leading anti-psychiatrist. I had seen one of the latter's patients, who I thought was in a hysterical stupor. The anti-psychiatrist was unhappy with my assessment, diagnosis and management.

\section{Case details}

Mrs G was a 64-year-old woman referred to my consultant. I admitted her to his unit in the hospital. He had telephoned and informed me that this was a well connected woman, very active socially but who lived on her own, as her husband, a highranking officer, was posted in Africa. For the last 2 days she had been sleeping (she had not got up nor had she eaten). She had been visiting a private psychiatrist in London for reasons unknown to the husband. She was not on any medication. The couple had no children. They lived in a mansion in the countryside. They enjoyed travelling abroad. Mrs G had a live-in domestic. She drank socially, did not smoke and enjoyed reading books on psychology, mental health, yoga and meditation. She enjoyed excellent health.

After admission, we telephoned her husband to ask about social and mental health issues. He told us that their marriage was happy, his wife 